\title{
Die europäischen Gewerkschaftsverbände. Zur Entwicklung ihrer Organisation und Politik seit den 1990er Jahren
}

\author{
Torsten Müller, Hans-Wolfgang Platzer und Stefan Rüb*
}

Der Prozess der europäischen Integration hat sich seit den 1990er Jahren in einer historisch beispiellosen Weise dynamisiert. Die Vertiefung und Erweiterung der Europäischen Union haben die Bedingungen der gewerkschaftlichen Interessenvertretung im Mehrebenensystem der Europäischen Union nachhaltig verändert und zugleich neue Anforderungen an eine transnationale Problembearbeitung gestellt:

- Durch die Einbindung einer großen Zahl neuer Mitgliedsverbände aus dem östlichen Europa in einem vergleichsweise kurzen Zeitraum ist die staatenübergreifende Gewerkschaftskooperation institutionell und politisch schwieriger geworden, da der Mitgliederzuwachs die (schon in der ,EU-15‘ vorhandene) ,Modellvielfalt', also die Varianz in den Strukturen und Praxen nationaler Arbeits- und Tarifbeziehungen erhöht hat und die Heterogenität der ökonomischen und arbeitspolitischen Mitgliederinteressen signifikant zugenommen hat.

- Durch die Binnenmarktvollendung, die umfassende Liberalisierung und Deregulierung der Märkte und die Etablierung der Währungsunion haben sich die Wettbewerbsverhältnisse im Integrationsraum weitreichend verändert und zu neuen Anforderungen einer transnationalen Koordination gewerkschaftlicher Arbeits- und Tarifpolitik und einer überstaatlich abgestimmten Interessenvertretung gegenüber den EU-Organen geführt.

Diese gleichzeitig zu bewältigenden Anforderungen eines komplexeren , management of diversity ' und eines anspruchsvolleren , management of interdependence "kennzeichnen mithin den Wandel der inneren und äußeren Verbandsumwelten der europäischen Gewerkschaftsverbände seit den 1990er Jahren.

In den politischen und wissenschaftlichen Debatten zum Themenfeld ,Gewerkschaften und EU-Integration' führen die transnationalen Branchenzusammenschlüsse der Gewerkschaften, die derzeit existierenden zwölf europäischen Gewerkschaftsverbände, die im Europäischen Gewerkschaftsbund (EGB) zusammengeschlossen sind und neben dessen nationalen Dachverbandsmitgliedern die ,zweite Säule‘ bilden, bislang eher ein Schattendasein. Auch in der mittlerweile umfangreichen Forschung zum Themenfeld, europäische Verbandspolitik und EU-Lobbyismus ' ${ }^{1}$ ist dieser Akteursgruppe, von älteren Arbeiten abgesehen, ${ }^{2}$ kaum Aufmerksamkeit geschenkt worden.

Dieser Befund erstaunt umso mehr, als die politische Ökonomie der EU-Integration (Währungsunion, Branchenliberalisierung, Industriepolitik) wie auch die regulatorischen Aktivitäten der Europäischen Union (darunter die Richtlinie zur Einrichtung Europäischer

* Dr. Torsten Müller, Forschungsgruppe europäische und globale Arbeitsbeziehungen an der Hochschule Fulda. Prof. Dr. Hans-Wolfgang Platzer, Jean Monnet Chair of European Integration, Hochschule Fulda.

Dr. Stefan Rüb, Forschungsgruppe europäische und globale Arbeitsbeziehungen an der Hochschule Fulda.

1 Zusammenfassend Rainer Eising/Beate Kohler-Koch: Interessenpolitik im europäischen Mehrebenensystem, in: Rainer Eising/Beate Kohler-Koch (Hrsg.): Interessenpolitik in Europa, Baden-Baden 2005, S. 11-75.

2 Ingrid Stöckl: Gewerkschaftsausschüsse in der EG, Kehl/Straßburg/Arlington 1986; Hans-Wolfgang Platzer: Gewerkschaftspolitik ohne Grenzen? Die transnationale Zusammenarbeit der Gewerkschaften im Europa der 1990er Jahre, Bonn 1991. 
Betriebsräte (EBR-Richtlinie ${ }^{3}$ ), Mitbestimmung in der Europäischen Aktiengesellschaft (SE-Richtlinie ${ }^{4}$ )) und die politisch-institutionellen Rahmensetzungen der Union (Sektorale Sozialdialoge) die Interessensphären und Vertretungsdomänen gerade der Branchengewerkschaften tangieren (vielfach in einem unmittelbareren und stärkeren Maße als die Vertretungsbereiche der gewerkschaftlichen Dachverbände). Es wäre mithin zu erwarten, dass sich sowohl die integrationswissenschaftliche Forschung, die sich Fragen der Europäisierung gesellschaftlicher Interessenvermittlung im Mehrebenensystem der Europäischen Union widmet, als auch die Gewerkschafts- und Arbeitsbeziehungsforschung, die nach der Herausbildung transnationaler Arenen der Arbeitsbeziehungen fragt, sich verstärkt dieses Gegenstands annehmen.

Angesichts dieses bislang brachliegenden Feldes der EU-Verbands- und Gewerkschaftsforschung ist dieser Beitrag, der auf Ergebnissen eines größeren Forschungsprojektes ${ }^{5}$ basiert, so angelegt, dass er einerseits einen knappen Überblick über maßgebliche organisationspolitische Entwicklungen gibt und andererseits eine zentrale analytische Problemstellung aufgreift, nämlich die Frage, ob und inwieweit die eingangs skizzierten weitreichenden Veränderungen der EU-Integrationsdynamik zu neuen Formen einer transnationalen Handlungskoordination und zu einem Wandel der Funktionsprofile der transnationalen Gewerkschaftsverbände geführt haben. Diese Themenstellung umfasst eine Fülle integrations-, verbands- und gewerkschaftstheoretischer Aspekte und kann im vorgegebenen Rahmen nur analytisch konturiert, empirisch illustriert und mit ersten generalisierbaren Befunden unterfüttert werden. Die für eine umfassende Analyse der gewerkschaftlichen Europäisierung notwendige Betrachtung der Entwicklungen im Bereich der nationalen Gewerkschaften ${ }^{6}$ muss ebenso ausgespart bleiben wie die Untersuchung gewerkschaftlicher Interessendurchsetzungsfähigkeit in konkreten Handlungsfeldern, die zugleich Teil der Frage nach den grundlegenden Veränderungen der gewerkschaftspolitisch relevanten Machtverhältnisse in der Europäischen Union ist. Um letzteren Debattenkontext nur anzudeuten, sei die aus einer gewerkschaftlichen Insiderperspektive formulierte Diagnose von Urban angeführt, wonach die wirtschaftliche Integrationslogik der vergangenen beiden Dekaden und die Machtverluste der Gewerkschaften eine , negative Symbiose ' bilden: „Die Festlegung nationaler Finanz- und Beschäftigungspolitik auf angebotspolitische Dogmen, der Ausfall der EZB-Geldpolitik für Wachstum und Beschäftigung, die Privatisierung öffentlicher Dienst- und Sozialleistungen sowie die direkte Infragestellung von Arbeitnehmer- und Gewerkschaftsrechten konstituieren ein politisch-ökonomisches Entwicklungsmodell, in dem die Machtressourcen des Kapitals zu- und die der organisierten Arbeit abnehmen. [...] Der Machtverlust der Gewerkschaften wird durch diese marktdominierte Integration vorangetrieben - und ist zugleich eine Erklärungsvariable dafür, dass es Europa an wirkungsmächtigen Korrekturen der Marktintegration fehlt."

3 Richtlinie 94/45/EG des Rates vom 22. September 1994 über die Einsetzung eines Europäischen Betriebsrats oder die Schaffung eines Verfahrens zur Unterrichtung und Anhörung der Arbeitnehmer in gemeinschaftsweit operierenden Unternehmen und Unternehmensgruppen, in: Amtsblatt der EG, Nr. L 254 vom 30. September 1994, S. 64-72.

4 Richtlinie 2001/86/EG des Rates vom 8. Oktober 2001 zur Ergänzung des Statuts der Europäischen Gesellschaft hinsichtlich der Beteiligung der Arbeitnehmer, in: Amtsblatt der EG, Nr. L 294 vom 10. November 2001, S. 22-32.

5 Hans-Wolfgang Platzer/Torsten Müller, unter Mitarbeit von Stefan Rüb/Matthias Helmer/Thomas R. Oettgen: Die globalen und europäischen Gewerkschaftsverbände. Handbuch und Analysen zur transnationalen Gewerkschaftspolitik, Berlin 2009.

6 Zur Transnationalisierung nationaler Gewerkschaften siehe Stefan Rüb: Die Transnationalisierung der Gewerkschaften. Eine empirische Untersuchung am Beispiel der IG Metall, Berlin 2009.

7 Hans-Jürgen Urban: Gewerkschaftliche Revitalisierung in einem neoliberalen und postmodernen Europa, in: Dieter Scholz u.a. (Hrsg.): „Europa sind wir“ - Gewerkschaftspolitik für ein anderes Europa, Münster 2009, S. 304-316, hier S. 304-305. 


\section{Organisationspolitische Entwicklungen: Arrondierung der transnationalen Organisa- tionslandschaft, Mitgliederexpansion und Ressourcenprobleme}

Im Folgenden sollen mittels Organisationsdaten ausgewählte Aspekte der organisationspolitischen Entwicklung der europäischen Gewerkschaftsverbände illustriert und beispielhaft einige der Probleme beleuchtet werden, die mit der geografischen Expansion und dem Mitgliederwachstum seit den 1990er Jahren verbunden sind.

\section{Tabelle: Organisationsdaten im Überblick}

\begin{tabular}{|c|c|c|c|c|}
\hline $\begin{array}{l}\text { Europäischer Gewerk- } \\
\text { schaftsverband }\end{array}$ & $\begin{array}{c}\text { Repräsentierte } \\
\text { Gesamt- } \\
\text { mitgliedschaft }\end{array}$ & $\begin{array}{l}\text { Mitglieds- } \\
\text { verbände }\end{array}$ & Länder & $\begin{array}{l}\text { Sekretariats- } \\
\text { sitz und } \\
\text { Personal }\end{array}$ \\
\hline $\begin{array}{l}\text { Europäischer Gewerk- } \\
\text { schaftsverband für den Öf- } \\
\text { fentlichen Dienst (EGÖD) }\end{array}$ & 8 Mio. & 209 & 36 & $\begin{array}{l}\text { Brüssel/ } \\
16 \text { Beschäftigte }\end{array}$ \\
\hline $\begin{array}{l}\text { Union Network Internatio- } \\
\text { nal-Europa (UNI-Europa) }\end{array}$ & 7 Mio. & 320 & 50 & $\begin{array}{l}\text { Brüssel/ } \\
18 \text { Beschäftigte }\end{array}$ \\
\hline $\begin{array}{l}\text { Europäisches Gewerk- } \\
\text { schaftskomitee für Bil- } \\
\text { dung und Wissenschaft } \\
\text { (EGBW) }\end{array}$ & 5,5 Mio. & 112 & 30 & $\begin{array}{l}\text { Brüssel/ } \\
10 \text { Beschäftigte }\end{array}$ \\
\hline $\begin{array}{l}\text { Europäischer Metallge- } \\
\text { werkschaftsbund (EMB) }\end{array}$ & 5,5 Mio. & $\begin{array}{l}72+ \\
4 \text { assoziierte }\end{array}$ & 33 & $\begin{array}{l}\text { Brüssel/ } \\
20 \text { Beschäftigte }\end{array}$ \\
\hline $\begin{array}{l}\text { Europäische Föderation der } \\
\text { Gewerkschaften für den } \\
\text { Lebensmittel-, Landwirt- } \\
\text { schafts- und Tourismussek- } \\
\text { tor (EFFAT) }\end{array}$ & 2,6 Mio. & 120 & 35 & $\begin{array}{l}\text { Brüssel/ } \\
11 \text { Beschäftigte }\end{array}$ \\
\hline $\begin{array}{l}\text { Europäische Transportar- } \\
\text { beiter-Föderation (ETF) }\end{array}$ & 2,5 Mio. & 223 & 40 & $\begin{array}{l}\text { Brüssel/ } \\
14 \text { Beschäftigte }\end{array}$ \\
\hline $\begin{array}{l}\text { Europäische Föderation der } \\
\text { Bergbau-, Chemie- und Ener- } \\
\text { giegewerkschaften (EMCEF) }\end{array}$ & 2,5 Mio. & 128 & 35 & $\begin{array}{l}\text { Brüssel/ } \\
8 \text { Beschäftigte }\end{array}$ \\
\hline $\begin{array}{l}\text { Europäische Föderation der } \\
\text { Bau- und Holzarbeiter } \\
(\mathrm{EFBH})\end{array}$ & 2,3 Mio. & 74 & 30 & $\begin{array}{l}\text { Brüssel/ } \\
8 \text { Beschäftigte }\end{array}$ \\
\hline $\begin{array}{l}\text { Europäischer Gewerk- } \\
\text { schaftsverband Textil, Be- } \\
\text { kleidung und Leder (EGV- } \\
\text { TBL) }\end{array}$ & 1 Mio. & 70 & 40 & $\begin{array}{l}\text { Brüssel/ } \\
4 \text { Beschäftigte }\end{array}$ \\
\hline $\begin{array}{l}\text { Europäische Föderation der } \\
\text { Polizei (EuroCOP) }\end{array}$ & 530.000 & 34 & 26 & $\begin{array}{l}\text { Luxemburg/ } \\
\text { 1-2 Beschäftigte }\end{array}$ \\
\hline $\begin{array}{l}\text { Europäische Allianz für } \\
\text { Kunst und Unterhaltung } \\
\text { (EAEA) }\end{array}$ & 300.000 & 135 & 27 & $\begin{array}{l}\text { Brüssel/ } \\
\text { kein eigenes } \\
\text { Sekretariat }\end{array}$ \\
\hline $\begin{array}{l}\text { Europäische Journalisten- } \\
\text { Föderation (EJF) }\end{array}$ & 280.000 & 53 & 34 & $\begin{array}{l}\text { Brüssel/ } \\
4 \text { Beschäftigte }\end{array}$ \\
\hline
\end{tabular}

Quelle: Eigene Zusammenstellung auf Basis der Datenerhebung von 2006 bis 2008. 
Betrachtet man die Entwicklung der gesamten transnationalen Organisationslandschaft der Gewerkschaften seit den 1990er Jahren, so ist zunächst die Neugründung der Europäischen Föderation der Polizei (EuroCOP) im Jahr 2002 zu nennen. Dieser Schritt hin zu einer transnationalen Organisation reflektiert die in den vergangenen Jahren erweiterten Kompetenzen und Aktivitäten der Europäischen Union im Bereich der inneren Sicherheit und folgt mithin einem Grundmuster europäischer Gewerkschaftsbildung, wonach die sektorale Gewerkschaftszusammenarbeit den ökonomischen und/oder politischen Vergemeinschaftungsprozessen in den jeweiligen Branchen folgt.

Aus Fusionen sind in den zurückliegenden zwei Jahrzehnten die Europäische Föderation der Bergbau-, Chemie- und Energiegewerkschaften (EMCEF, 1996) und die Union Network International-Europa (UNI-Europa, 2000) hervorgegangen. In beiden Fällen wurde die europäische Formierung zeitgleich und strukturanalog zu entsprechenden branchengewerkschaftlichen Fusionen auf globaler Verbandsebene vollzogen. Die Fusionen sind Ressourcenzwängen und Bestandssicherungsinteressen kleinerer, ehemals eigenständiger transnationaler Organisationen geschuldet, wobei die Form der transnationalen Reorganisation in starkem Maße durch vorangegangene Fusionen im Bereich dominanter nationaler Mitgliedsgewerkschaften (vergleicht man etwa die Matrixstruktur von UNI-Europa mit der von Ver.di) geprägt wird. Der historische Langzeittrend des Zusammenschlusses ehemals eigenständiger transnationaler Sektorenorganisationen zu Multi-Branchengewerkschaften wird sich, nach aller Voraussicht, fortsetzen. Beispielsweise laufen seit 2009 Sondierungsgespräche zwischen dem Europäischen Metallgewerkschaftsbund (EMB), der EMCEF und dem Europäischen Gewerkschaftsverband Textil, Bekleidung und Leder (EGV-TBL), die darauf zielen, die Ressourcen der transnationalen Sekretariate und die innerverbandlichen Arbeitsstrukturen enger (wenn auch zunächst unterhalb der vollständigen Fusion) zu verzahnen.

Schließlich repräsentieren die Europäische Transportarbeiter-Föderation (ETF, 1999) und die Europäische Allianz für Kunst und Unterhaltung (EAEA, 2001) eine Entwicklung, die auch die Entstehung, älterer' europäischer Verbände kennzeichnet, nämlich die statuarische und politische Verselbstständigung der europäischen Organisationsebene gegenüber dem jeweiligen globalen Verband. Mit diesen Schritten der ETF und der EAEA ist organisationspolitisch bei allen derzeit existierenden europäischen Gewerkschaftsverbänden ein bis in die Anfangsjahre der EU-Integration zurückreichender Prozess der ,Emanzipation ' der europäischen von der jeweils globalen Verbandsebene abgeschlossen.

Alle europäischen Gewerkschaftsverbände sind, sieht man von der Neugründung der EuroCOP (2002) ab, seit den 1990er Jahren in ihrer Gesamtmitgliedschaft, in der Zahl der angeschlossenen Bünde und in der geografischen Reichweite der Herkunftsländer gewachsen.

Außer der Europäischen Föderation der Bau- und Holzarbeiter (EFBH), der EAEA und der EuroCOP, deren Mitgliederkreis sich auf die EU-Mitgliedstaaten beschränkt, repräsentieren die anderen europäischen Gewerkschaftsverbände inzwischen über die Europäische Union hinausgreifende Mitgliederkreise, die im Falle der UNI-Europa mit 50 Herkunftsländern sogar eine paneuropäische Reichweite haben.

Ein mit dem Ende der Teilung Europas begonnener, stufenweiser Prozess der Assoziierung und Integration der sich transformierenden beziehungsweise neu gründenden Gewerkschaften aus den Ländern des ehemaligen Ostblocks ist mittlerweile bei allen europäischen Verbänden zum Abschluss gekommen. Einer erfolgreich vollzogenen,formalen“ Integration der mittel- und osteuropäischen Gewerkschaften stehen bei nahezu allen Verbänden weiterhin schwierige Anforderungen und teilweise ungelöste Probleme einer , substanziellen` Integration der neuen Mitgliedsorganisationen gegenüber. Diese Diskrepanzen sind zunächst ei- 
nem Ressourcenproblem geschuldet: Zum einen haben die Gewerkschaften in den mittelund osteuropäischen Ländern bei ohnehin begrenzten finanziellen Ressourcen seit Anfang 2000 mehrheitlich deutliche Mitgliederrückgänge zu verzeichnen. Ihre schwache Finanzausstattung begrenzt ihre Möglichkeiten zu einer regelmäßigen und umfassenden Beteiligung an den transnationalen verbandlichen Arbeitsprozessen. Zum anderen hat der Erweiterungsprozess die strukturellen Ressourcenprobleme aller transnationalen Verbände verschärft. Trotz wiederholter (moderater) Beitragserhöhungen, die nahezu alle europäischen Föderationen in den vergangenen Jahren vorgenommen haben, hat die finanzielle Ausstattung nicht mit den gestiegenen Anforderungen der Integration eines größeren und heterogeneren Mitgliederkreises Schritt gehalten. Entsprechend stark hat im operativen Bereich - insbesondere im Bereich der mitgliederbezogenen Politik (Schulung, Beratung, Erstellung von Studien etc.) - die Abhängigkeit der meisten europäischen Verbände von ,externen' Mitteln zugenommen. Dabei handelt es sich überwiegend um (Projekt-)Mittel der Europäischen Union. Die Finanzierung der Arbeit aller europäischer Gewerkschaftsverbände aus EU-Mitteln ist beträchtlich und bewegt sich zwischen 20 und 60 Prozent des jeweiligen Gesamtbudgets der transnationalen Organisationen.

Die Mitgliederexpansion - bei zahlreichen Verbänden handelt es sich um eine Verdoppelung der Mitgliedsorganisationen in zwei Jahrzehnten - hat neben den prekären Ressourcenentwicklungen zugleich die innerverbandlichen Voraussetzungen der transnationalen Handlungskoordination deutlich verändert und tendenziell erschwert: institutionell durch die gewachsene Vielfalt der zu vermittelnden gewerkschaftlichen Identitäten und Praxen, politisch unter anderem durch die begrenzten Fähigkeiten der Gewerkschaften in den mittel- und osteuropäischen Ländern zu einer national-dezentralen Um- und Durchsetzung der transnational vereinbarten Politiken. So erschweren beispielsweise die mehrheitlich schwach entwickelten betrieblichen Strukturen der Interessenvertretung und das (mit Ausnahme Sloweniens und teilweise Ungarns und der Slowakei) weitgehende Fehlen eines überbetrieblichen, branchenbezogenen Systems autonomer Tarifbeziehungen ${ }^{8}$ die Kooperationsansätze der transnationalen Verbände in den zentralen Feldern der Konzernpolitik (EBR) und der transnationalen Tarifkoordination.

Von Beginn der Transformationsprozesse an bis zur Gegenwart dien(t)en die europäischen Föderationen primär als Foren des Informationsaustausches zwischen westlichen und östlichen Mitgliedsgewerkschaften und als eine flankierende Ebene zur Vermittlung von Dienstleistungen (Beratung, Qualifizierung, Heranführung der neuen Mitglieder an die europäischen Strukturen etc.). Zu keinem Zeitpunkt waren die europäischen Gewerkschaftsverbände der ,Ort‘ einer strategischen Steuerung des Erweiterungsprozesses. Die vorherrschenden Kooperationsmuster waren (und sind) bilaterale oder regional zugeschnittene ,Patenund Partnerschaften', die von den nationalen Gewerkschaften aus den westeuropäischen Ländern getragen werden. Diese Prozesse waren anfangs der Transformationsperiode teilweise auch von einem Konkurrenzverhältnis unter den nationalen Gewerkschaften aus den ,alten` EU-Mitgliedstaaten um einen jeweils nationalen ,Modellexport' geprägt.

Greift man unter der Vielzahl der für die Analyse der Organisationspolitik relevanten Parameter (darunter die Entwicklung der innerverbandlichen Willensbildungsstrukturen und Entscheidungsverfahren, der Ausschüsse und Beratungsgremien) an dieser Stelle die trans-

8 Heribert Kohl/Hans-Wolfgang Platzer: Industrial Relations in Central and Eastern Europe. Transformation and Integration. A comparison of the eight new EU member states, Brüssel 2004; Heribert Koh1/Hans-Wolfgang Platzer: West-East Trade Union Cooperation and Integration in the New Europe: Barriers, Challenges and Opportunities, in: Otto Jacobi u.a. (Hrsg.): Social Embedding and the Integration of Markets. An Opportunity for Transnational Trade Union Action or an Impossible Task?, Düsseldorf 2007, S. 193-215. 
nationalen Sekretariate heraus, die die Gesamtorganisation in den alltäglichen Arbeitsprozessen , auf Brüsseler Ebene" repräsentieren, so verdienen zwei Befunde hervorgehoben zu werden. Zum einen beschäftigt nur die Hälfte aller Verbände mehr als zehn Mitarbeiter. Zum anderen wurden bei der Mehrzahl der Verbände die transnationalen Sekretariate während der beiden vergangenen Dekaden zwar personell leicht aufgestockt, bei keinem Verband aber umfassend ausgebaut. Würde man die Entwicklung der Ressourcen und gegenwärtigen personellen Ausstattung der europäischen Sekretariate zum alleinigen Maßstab der Beurteilung der Transnationalisierung und Europäisierung gewerkschaftlicher Politik machen, wären die Befunde insgesamt sehr ernüchternd. Eine Beurteilung der Ressourcenausstattung der Sekretariate der europäischen Sektorengewerkschaften muss jedoch mehrere Faktoren in Rechnung stellen:

- Bestimmte Regulierungsaktivitäten der Europäischen Union sind branchenübergreifender Natur und werden im Rahmen einer gewerkschaftlichen Arbeitsteilung vom Dachverband, dem EGB, und dessen fachlich-wissenschaftlicher Infrastruktur, dem Europäischen Gewerkschaftsinstitut, abgedeckt.

- Ferner sind, wie unten weiter ausgeführt wird, nicht allein die finanziellen und personellen Sekretariatskapazitäten für eine effektive Interessenvertretung ausschlaggebend, sondern die Intensität, mit der sich die nationalen Mitgliedsverbände an der transnationalen $\mathrm{Zu}-$ sammenarbeit beteiligen und ihr Know-how und ihre (personellen) Ressourcen in die (Gremien-)Arbeit der europäischen Verbände einbringen.

Gleichwohl bleiben die transnationalen Kapazitätsprobleme der Sekretariate schon deshalb virulent, weil sich im Vergleich zu den unternehmerischen Vertretungsstrukturen auf europäischer Ebene seit jeher gravierende Asymmetrien auftun und weil diese Ungleichgewichte zwischen der Vertretung von Kapitalinteressen und der Repräsentation von Arbeitnehmerinteressen im Zuge der Binnenmarktvollendung weiter zugenommen haben. ${ }^{9}$

Die Ressourcenprobleme und Herausforderungen einer angemessenen transnationalen Interessenrepräsentation beschreibt Schmidt-Hullmann aus einer praktischen Erfahrungsperspektive (als Leiter der Europaabteilung der deutschen IG-BAU) wie folgt: ,[...] wir bleiben unter unseren Möglichkeiten. Wenn es hoch kommt beschäftigen wir eine niedrige dreistellige Zahl von GewerkschaftsvertreterInnen in Brüssel. Beim EGB decken wenige Menschen alle Politikfelder ab und davon gibt es in Brüssel jede Menge. In den europäischen Branchenbünden ist die Lage nicht besser. Um überhaupt noch halbwegs präsent sein zu können, begibt man sich in die finanzielle Abhängigkeit von Kommissionsmitteln, um strukturelle Aufgaben und einen Teil des Personals zu finanzieren. [...] Mögliche Abhilfe: Strategische Entscheidung für eine deutliche Erhöhung der Aufwendungen für die Interessenvertretung auf europäischer Ebene mit dem Ziel, die dafür notwendigen strukturellen Ausgaben aus Eigenmitteln zu finanzieren. Alternativ: Bildung elektronischer europaweiter Netzwerke und/ oder befristete Bildung von Kampagnengruppen in Brüssel aus entsandten Hauptamtlichen der Mitgliedsbünde zu wichtigen Themen." 10

Ungeachtet dieser in der gewerkschaftsinternen Debatte zurecht reklamierten Bedarfe im Bereich der Ressourcen und strategischen Orientierungen zeigen sich im historischen Längsschnitt betrachtet - und bei allen Unterschieden zwischen den zwölf europäischen Ge-

9 Quantitative Befunde zur Vertretungsdichte unterschiedlicher gesellschaftlicher Interessen auf EU-Ebene finden sich in Eising/Kohler-Koch: Interessenpolitik im europäischen Mehrebenensystem, 2005.

10 Frank Schmidt-Hullmann: Was können und was müssen Gewerkschaften europapolitisch tun, um erfolgreich zu werden?, in: Dieter Scholz u.a. (Hrsg.): „Europa sind wir“- Gewerkschaftspolitik für ein anderes Europa, Münster 2009, S. 242-252, hier S. 248. 
werkschaftsverbänden ${ }^{11}$ - doch Entwicklungen, die Fortschritte in der Europäisierung und Transnationalisierung gewerkschaftlicher Politik markieren und sich wie folgt verallgemeinern lassen:

Das im Zuge der vertieften EU-Marktintegration komplexere und anspruchsvollere ,management of interdependence ' gewerkschaftlicher Interessen und die im Zuge der EU-Osterweiterung gleichzeitig gewachsenen und schwierigeren Anforderungen eines ,management of diversity“ haben bei keinem europäischen Gewerkschaftsverband dazu geführt, auf transnationaler Sekretariatsebene die personellen Ressourcen nachhaltig zu stärken und die Kompetenzen zu erweitern oder gar insgesamt die Entscheidungsprozesse auf europäischer Ebene zu zentralisieren. Kein europäischer Gewerkschaftsverband orientiert sich demzufolge an einem ,Hierarchiemodell'.

Vielmehr dominiert bei allen europäischen Gewerkschaftsverbänden ein Handlungsansatz, der sich (mit einer gewissen Zuspitzung) als ,Arbeitsgruppenmodell ‘ beschreiben lässt. Demnach sind die von nationalen Gewerkschaftsexperten getragenen Ausschüsse oder ,task forces', die sich (sub-)sektoralen Fragen beziehungsweise Querschnittsaufgaben widmen, die zentralen Organisations- und Arbeitseinheiten der transnationalen Verbände. Die Ausdifferenzierung dieser innerverbandlichen Willensbildungsstrukturen, die Verdichtung der in diesen Gremien laufenden Kommunikations- und Abstimmungsprozesse und ein tendenziell höherer Verbindlichkeits- und Verpflichtungsgrad der getroffenen Entscheidungen sind wesentliche Charakteristika der transnationalen Verbandsentwicklungen seit den 1990er Jahren.

\section{Zur transnationalen Interessenvermittlung der europäischen Gewerkschaftsver- bände: zwischen traditioneller ,labour diplomacy ${ }^{6}$ und neuen Handlungsansätzen}

Bei der Frage, welche Rolle die europäischen Gewerkschaftsverbände in der Vermittlung von Mitgliederinteressen spielen (sollten), taucht wiederholt ein grundlegendes Problem zwischen ,Mitgliederlogik ' und ,Einflusslogik ${ }^{6} 2$ auf, das Hyman wie folgt beschreibt: „Indeed trade unionism at any level involves a delicate interaction between the tasks of expressing the views and aspirations of those represented, and adapting the form and content of their representation to the preferences of the counterparts in negotiation. But at supranational level, simply because the distance of representatives from the membership is greater, and the resources available are often more limited than at national level, the logic of influence typically acquires greater force. This logic can easily push unions into a mode of 'labour diplomacy " $[\ldots]$ which distances them from their constituencies and strengthens their dependence on acceptance by their interlocutors". ${ }^{13}$

In der Tat sind die gewerkschaftlichen Handlungsformen und die Wege und Instrumente der gewerkschaftlichen Interessenvertretung auf europäischer Ebene in starkem Maße durch die institutionelle Logik des EU-Systems, vorstrukturiert'. Ein entsprechend hohes Gewicht kommt der , labour diplomacy ‘ in Gestalt des institutionalisierten beziehungsweise informellen Lobbying zu. Allerdings variiert diese Einflussform nach einzelnen Branchen beträchtlich und zwar je nach dem, wie ausgeprägt die jeweiligen sektoralen EU-Industriepolitiken sind und wie stark die supranationale Steuerungshoheit bei der Festlegung branchenrelevan-

11 Vgl. hierzu im Einzelnen Platzer/Müller: Die globalen und europäischen Gewerkschaftsverbände, 2009.

12 Jon Eric Dølvik: Redrawing Boundaries of Solidarity? ETUC, social dialogue and the Europeanisation of trade unions in the 1990s, Oslo 1997; Franz Traxler/Philippe C. Schmitter: The Emerging Euro-Polity and Organized Interests, in: European Journal of Industrial Relations 2/1995, S. 191-218.

13 Richard Hyman: Labour, Markets and the Future of 'Decommodification', in: Otto Jacobi u.a. (Hrsg.): Social Embedding and the Integration of Markets. An Opportunity for Transnational Trade Union Action or an Impossible Task?, Düsseldorf 2007, S. 11-30, hier S. 22-23. 
ter Parameter ist (etwa im Bereich der Europäischen Föderation der Gewerkschaften für den Lebensmittel-, Landwirtschafts- und Tourismussektor (EFFAT) die EU-Agrarmarktordnungen oder im Bereich der EMCEF die EU-Chemikaliengesetzgebung). Während beispielsweise der EMB in den Metallsektoren Stahl und Schiffbau auf eine strategisch ausgerichtete EU-Industriepolitik trifft und als Akteur in die supranational institutionalisierten Konzertierungen einbezogen ist, herrschen in den Dienstleistungssektoren Gesetzgebungsaktivitäten der Europäischen Union vor, die auf die Liberalisierung der Märkte zielen, ohne dass es etwa für die UNI-Europa, den Europäischen Gewerkschaftsverband für den Öffentlichen Dienst (EGÖD) oder die EAEA eine vergleichbar institutionalisierte Steuerung durch die EU-Kommission nach (Sub-)Sektoren oder Berufsgruppen gäbe.

Die Frage nach den Möglichkeiten und Grenzen gewerkschaftlicher Interessendurchsetzung mit den Mitteln der , labour diplomacy ' kann hier nicht empirisch vertieft, sondern allenfalls angedeutet werden. Grundsätzlich hat die Vertiefung der europäischen Integration der letzten zwanzig Jahre, die auf einem wirtschaftsliberalen Ansatz basierte und vorrangig durch die Schaffung von Handels- und Kapitalfreiheit und die Deregulierung von Märkten geprägt ist, ${ }^{14}$ die gewerkschaftliche Interessenvermittlung auf nationaler und europäischer Ebene strukturell erschwert. In der gegenwärtigen Europäischen Union ist das Verhältnis von ökonomischer und politisch-sozialer Integration asymmetrisch. Einer supranationalen Wettbewerbsordnung und einem supranationalen Währungsregime stehen nur sehr begrenzt supranationale und mehrheitlich intergouvernementale Verfahren der Regulierung und Steuerung in den Feldern der Wirtschafts-, Beschäftigungs- und Sozialpolitik gegenüber. ${ }^{15}$ Im Rahmen einer solchen primär auf die Wettbewerbsfähigkeit Europas abzielenden ,Liberalisierungsgemeinschaft" ${ }^{*}$ befinden sich die Gewerkschaften in einer defensiven Position, in der es primär darum geht, die negativen Auswirkungen der Liberalisierungspolitik abzuschwächen beziehungsweise zu verhindern.

Die im Dezember 2006 verabschiedete Dienstleistungsrichtlinie ${ }^{17}$ ist in jüngster Zeit das wohl prominenteste Beispiel einer Gesetzgebungsinitiative, bei der es den Gewerkschaften gelang, unerwünschte Auswirkungen abzuwehren und mittels einer zwischen nationaler und europäischer Ebene kohärent koordinierten Mehrebeneneinflusspolitik, die neben dem Lobbying zunehmend auch, klassische' gewerkschaftliche Mobilisierungsmittel wie Warnstreiks und Demonstrationen umfasst, in ihrem Sinne zu beeinflussen. ${ }^{18}$ Die im Dezember 2008 erfolgte Revision der EBR-Richtlinie ist ein weiteres Beispiel eines Gesetzgebungsprozesses der Europäischen Union, bei dem es den europäischen Gewerkschaften gelang, zumindest einen Teil ihrer Forderungen und Interessen durchzusetzen. Vereinzelt, wie zum Beispiel im Falle der Entsenderichtlinie, ${ }^{19}$ sind die Gewerkschaften darüber hinaus in der

14 Fritz W. Scharpf: Regieren in Europa. Effektiv und demokratisch?, Frankfurt Main 1999.

15 Hans-Wolfgang Platzer: Europäisches Sozialmodell und Arbeitsbeziehungen in der erweiterten EU. Ein Problemaufriss, in: Alexandra Baum-Ceisig/Anne Faber (Hrsg.): Soziales Europa? Perspektiven des Wohlfahrtsstaates im Kontext von Europäisierung und Globalisierung, Wiesbaden 2005, S. 152-182.

16 Wolfgang Streeck: Europäisierung als Liberalisierung: Perspektiven gewerkschaftlicher Politik im integrierten Europa, in: Wolfgang Streeck (Hrsg.): Korporatismus in Deutschland. Zwischen Nationalstaat und Europäischer Union, Frankfurt Main 1999, S. 41-66, hier S. 50.

17 Richtlinie 2006/123/EG des Europäischen Parlaments und des Rates vom 12. Dezember 2006 über Dienstleistungen im Binnenmarkt, in: Amtsblatt der EG, Nr. L 376 vom 27. Dezember 2006, S. 36-68.

18 Lisa Maria Arnold: Die Entstehung der europäischen Dienstleistungsrichtlinie im Spannungsfeld organisierter Interessen: Eine Fallstudie zum Einfluss von Gewerkschaften und Unternehmerverbänden im Europäischen Parlament, Hertie School of Governance - working papers Nr. 36, Berlin 2008; Wolfgang Kowalsky: Outcome of the battle on the Services Directive, in: Transfer 1/2007, S. 147-149.

19 Richtlinie 96/71/EG des Europäischen Parlaments und des Rates vom 16. Dezember 1996 über die Entsendung von Arbeitnehmern im Rahmen der Erbringung von Dienstleistungen, in: Amtsblatt der EG, Nr. L 18 vom 21. Januar 1997, S. 1-6. 
Lage ihrerseits Regulierungsaktivitäten der Europäischen Union anzustoßen, indem sie einen gesetzgeberischen Handlungsbedarf begründen und Lösungsvorschläge durch gezieltes Lobbying gegenüber den Entscheidungsorganen der Europäischen Union vermitteln.

Nicht zuletzt als Ergebnis längerfristiger gewerkschaftlicher Bemühungen sind auf europäischer Ebene in Ansätzen Arenen transnationaler Arbeitsbeziehungen entstanden. Dies sind zum einen die Ausschüsse des Sektoralen Sozialdialogs (ASSD), deren Einrichtung gewerkschaftlichen Forderungen Rechnung trägt. Diese durch die EU-Kommission gesteuerten und von ihr mit politischen Anreizen (darunter die ,Anerkennung' der Verbände als ,repräsentative“ Akteure) versehenen Dialogstrukturen ,zwingen“ die sektoralen Unternehmensverbände in die Rolle von Arbeitgeberorganisationen und verschaffen den europäischen Gewerkschaftsverbänden mithin einen ,Gegnerbezug ‘.

Dies sind zum anderen transnationale konzernpolitische Arenen, deren Entwicklung durch entsprechende Regulierungsaktivitäten der Europäischen Union (EBR- und SE-Richtlinie) befördert wurde und die den europäischen Gewerkschaftsverbänden gleichfalls neue Handlungsoptionen eröffnen.

In diesen Handlungsfeldern tritt eine zweite, die , labour diplomacy “ ergänzende Form der Interessenvermittlung durch die europäischen Gewerkschaftsverbände hinzu: auf Konzernebene die Intensivierung von transnationalen Dialogbeziehungen mit dem Management und auf sektoraler Ebene die Entwicklung von ersten Ansätzen einer bilateralen transnationalen Verhandlungs- und Vereinbarungspolitik mit der Arbeitgeberseite. Im historischen Längsschnitt der gesamten Entwicklung der Europäischen Union und der europäischen Gewerkschaftszusammenarbeit seit den 1950er Jahren betrachtet, stellen diese Entwicklungen ein Novum dar. Das heißt, das ,Verhandeln und Vereinbaren', eine historische Kernfunktion der Gewerkschaften und bislang eine rein nationalstaatliche Domäne, hat in einigen Fällen eine transnationale Dimension angenommen. Diese Entwicklung hat dazu geführt, dass die europäischen Verbände zusätzliche Handlungsfelder besetzt und Instrumente entwickelt haben, die bei einzelnen Verbänden, wie etwa bei der ETF im Bereich des Sektoralen Sozialdialogs, inzwischen gleichgewichtig neben der , labour diplomacy' und der lobbyistischen Interessenvermittlung stehen.

Ein drittes Handlungsfeld, das weder europarechtlich abgesichert noch durch institutionelle Anreize der EU-Kommission flankiert ist, ist die Tarifpolitik. Im Bereich der Lohnund Tarifpolitik sind die Gewerkschaften unter den Bedingungen der Währungsunion zu einer unilateralen transnationalen Steuerung und Selbstregulierung gezwungen, wenn sie problematische lohnpolitische Verwerfungen und damit Standortkonflikte begrenzen oder verhindern wollen.

Somit kann die Gesamtentwicklung der Handlungs- und Einflussformen der europäischen Gewerkschaftsverbände dahingehend generalisiert werden, dass neben die integrationsgeschichtlich lange Zeit vorherrschende, labour diplomacy ' seit den 1990er Jahren zwei weitere Handlungsformen getreten sind, die transnationale ,tarifautonome ' Vereinbarungspolitik auf Konzern- und sektoraler Ebene und die transnationale gewerkschaftliche, Selbsthilfe' im Bereich der Tarifpolitik.

Diese nach Verbänden und deren sektoralen Handlungsbedingungen in unterschiedlichen Mischungsverhältnissen auftretenden Handlungsformen lösen das eingangs zitierte Spannungsverhältnis zwischen dezentraler , mitgliedstaatlicher Basis “ und supranationaler, europäisch abgehobener Gewerkschaftsdiplomatie` zwar nicht grundsätzlich auf, relativieren es aber insofern, als diese neuen Politikansätze die grenzübergreifende Vernetzung und Interaktion eines breiteren Kreises von nationalen gewerkschaftlichen Akteuren erfordern und befördern. 
Die Intensivierung und Verdichtung dieser horizontal-grenzübergreifenden Interaktionen wie auch der vertikalen Interaktionen zwischen europäischer Verbands- und Sekretariatsebene und nationalen gewerkschaftlichen Funktionsbereichen (Unternehmenspolitik, Tarifpolitik) ist mithin ein prägendes Kennzeichen und Entwicklungsmerkmal der transnationalen Verbände der jüngeren Vergangenheit.

\section{Zur Erweiterung der Aufgabenbereiche und zum Wandel der Funktionsprofile der europäischen Gewerkschaftsverbände}

Will man vor dem Hintergrund der eingangs skizzierten organisationspolitischen Entwicklungen und der oben knapp illustrierten Handlungsmuster die Frage beantworten, welche Funktionen die transnationalen Verbände für ihre Mitglieder im Rahmen einer europäischen gewerkschaftlichen Mehrebenenpolitik erfüllen und ob und inwieweit eine Erweiterung der Aufgabenbereiche und eine Intensivierung der Koordinierungsprozesse stattgefunden hat, die auf einen Wandel der Funktionsprofile insgesamt schließen lassen, sind die sektorale Dialogpolitik, die Tarifpolitik und die Unternehmenspolitik zentrale empirische Beobachtungsfelder. Denn zum einen sind dies die Handlungsfelder, in denen sich seit gut einer Dekade neue transnationale Arenen und Problemlösungsansätze herausgebildet haben, und zum anderen sind dies Felder, die traditionell zu den Kernaufgaben der nationalen Branchengewerkschaften gehören. Ein knapper, mit empirischen Beispielen unterfütterter Überblick mag diese Entwicklungen andeuten.

Die ASSD, deren Zahl sich gegenwärtig auf 39 beläuft, schaffen den institutionellen Rahmen für eine Co- oder Selbstregulierung der sektoralen Sozialpartnerverbände. Die vergleichende Analyse der bisherigen Politikergebnisse zeigt, dass die ASSD allenfalls notwendige, aber keine hinreichenden Voraussetzungen für eine substanzielle transnationale Vereinbarungspolitik schaffen. Angesichts des grundsätzlich voluntaristischen Charakters des Sektoralen Sozialdialogs und der fehlenden Druckmittel der Gewerkschaften bestimmt die weniger ambitionierte Zielsetzung der Arbeitgeberseite das Tempo der Entwicklung und die Ergebnisse. Auch die Kommission kann als ,Prozessmanagerin' lediglich über eine entsprechende Gestaltung der strukturellen, politischen und materiellen Rahmenbedingungen Anreize setzen. Unter diesen Voraussetzungen sind die europäischen Gewerkschaftsverbände bislang nicht in die Rolle einer die nationale Ebene systematisch ergänzenden oder gar ersetzenden ,transnationalen Kollektivvertragspartei ' hineingewachsen. Mit dieser Entwicklungsoption ist auch in voraussehbarer Zukunft nicht zu rechnen.

Dennoch ist die Bedeutung der europäischen Verbandsebene als ,Ort' einer verdichteten zwischengewerkschaftlichen Kommunikation und Handlungskoordination und die der Sekretariate beziehungsweise Generalsekretäre als ,Repräsentanten“ in den ASSD-Prozessen tendenziell gewachsen, da auch die , weichen "Verhandlungsgegenstände eine innerverbandliche Interessenabklärung auf europäischer Ebene erfordern und der transnationale Verband (fallweise) mandatiert werden muss. Angesichts einer vergleichsweise kurzen Entwicklungsphase zahlreicher ASSD sind allerdings die für eine erfolgreiche Implementierung notwendigen , level linkages' zwischen nationaler und europäischer Ebene vielfach noch nicht hinreichend entwickelt.

Die europäischen Gewerkschaftsverbände messen dem Sektoralen Sozialdialog eine nach Branchenbedingungen variierende politisch-strategische Bedeutung bei. Vor allem die ASSD in den (Sub-)Sektoren, die stark von legislativen Aktivitäten auf europäischer Ebene betroffen sind, haben für die Gewerkschaften ein besonderes strategisches Gewicht. Nicht selten dient der Sektorale Sozialdialog auch als Rahmen, in dem die Gewerkschaften versu- 
chen, zusammen mit der Arbeitgeberseite gemeinsame industriepolitische Brancheninteressen abzustimmen und diese als Teil ihres Lobbying in die europäischen Entscheidungsprozesse einzubringen.

Schließlich spielen die ASSD bei allen europäischen Gewerkschaftsverbänden unter logistischen, konzeptionellen und finanziellen Gesichtspunkten eine beträchtliche Rolle. Denn die Anreizmechanismen, die die EU-Kommission bereithält (Räumlichkeiten, Dolmetscherfunktionen, Reisekosten, Gelder für sektorale Studien etc.), werden von den europäischen Gewerkschaftsverbänden systematisch als Ressource ihrer mitgliederbezogenen Aktivitäten genutzt. Alles in allem führen die ASSD-Prozesse zu einer, formalen ' Anerkennung und Aufwertung des Akteursstatus der europäischen Gewerkschaftsverbände. Bei Abstimmungsprozessen im Bereich einer europäischen sektoralen Arbeitspolitik wächst die europäische Verbandsebene über eine reine Forumsfunktion (Erfahrungsaustausch, verständigungsorientierte Annäherung, unverbindliche Entscheidungen) hinaus, die noch bis in die 1980er Jahre hinein die meisten Verbände prägte. Freilich wird dieses erweiterte Profil, das sich in , verbindlichen ' Zielfestlegungen und engeren Steuerungsprozessen auf transnationaler Ebene manifestiert, dadurch relativiert, dass dem enormen quantitativen Wachstum gemeinsamer Stellungnahmen oder Rahmenabkommen eine nach wie vor geringe materielle Substanz der Vereinbarungsinhalte gegenübersteht. Lediglich in den (bislang) wenigen Fällen einer substanziellen Vereinbarungspolitik (wie im Verkehrssektor) wird der transnationale Verband zum , clearinghouse ' der (durchaus unterschiedlichen) Interessen der nationalen Mitgliedsverbände und zu einer genuin europäischen Steuerungsebene.

Angesichts einer weiter fortgeschrittenen Verflechtung der europäischen Ökonomien im Zuge von Binnenmarkt und Währungsunion in den 1990er Jahren ist auch im Handlungsfeld der transnationalen Tarifkoordination eine gestiegene Bereitschaft der nationalen Mitgliedsverbände erkennbar, sich stärker in Koordinierungsprozesse unter dem Dach der europäischen Gewerkschaftsverbände einbinden zu lassen. Dieser Politikansatz hat zugleich bei den meisten Verbänden zu einer Ausweitung und Ausdifferenzierung der Beratungs- und Willensbildungsstrukturen auf der europäischen Verbandsebene geführt. Die europäischen Gewerkschaftsverbände treten über die verbandlich getroffenen Absprachen über Lohnformeln, Verhandlungsleitlinien und Verhandlungsgegenstände als virtuelle Koordinierungsinstanz auf. Sie sind aber weder im Vorfeld noch in den laufenden Prozess der Tarifverhandlungen ihrer Mitgliedsgewerkschaften direkt eingebunden. Sie sind günstigstenfalls von ihren Mitgliedsgewerkschaften mit der Kompetenz ausgestattet, das Tarifverhalten der nationalen Mitglieder auf die Konformität mit dem Koordinierungsansatz hin zu evaluieren, nicht jedoch mit harten Sanktionsmöglichkeiten bei etwaigen Verstößen eines nationalen Mitgliedsverbands gegen die gemeinsamen Absprachen. Die europäischen Gewerkschaftsverbände erfüllen im Bereich der europäischen Tarifkoordinierung gleichwohl wichtige Funktionen in den Bereichen des Prozessmanagements, der Kommunikation und (teilweise) der Konfliktmoderation sowie der Organisation. Diese Funktionen sind allerdings nach einzelnen Verbänden unterschiedlich stark ausgeprägt beziehungsweise bislang unterschiedlich weit entwickelt. Insgesamt zeigt sich ein Entwicklungsmuster der ,Nachahmung bewährter Praxis‘. Das heißt, die im Bereich der europäischen Branchenföderationen vom EMB zuerst entwickelten innerverbandlichen Willensbildungsstrukturen und Verfahrensregeln zur transnationalen Tarifkoordination wurden nach und nach (zumindest in den Grundzügen) auch von anderen Verbänden übernommen, wobei die EFBH darüber hinaus im Bereich staatenübergreifender Bauprojekte neue Ansätze einer , direkten ' transnationalen Lohnpolitik verfolgt. 
Bei einzelnen Verbänden, darunter dem EMB als Vorreiter der transnationalen Tarifkoordination, sind Entwicklungen erkennbar, wonach die ,moralische Selbstverpflichtung ' darauf zielt, den Verbindlichkeitsgrad der tarifpolitischen Koordinierung durch einen wechselseitigen Legitimationsdruck zu steigern. Dazu dienen vor allem die fortlaufende Erfassung und Verfügbarmachung sowie die regelmäßige Bilanzierung der tarifpolitischen Ergebnisse im Rahmen verschiedener Netzwerke und transnationaler Kommunikationsräume (tarifpolitischer Ausschuss, regionale Tarifpartnerschaften, tarifpolitische Konferenzen, Schulungsseminare etc). Insgesamt hat die transnationale Tarifkoordination zu einer Funktionserweiterung und -aufwertung der europäischen Gewerkschaftsverbände geführt. Bewegte sich deren tarifpolitisches Funktionsprofil noch bis Anfang der 1990er Jahre auf dem Niveau eines Forums des Informationsaustausches, so haben sich mittlerweile durch die Verständigung auf (, verbindliche") tarifpolitische Leitlinien und/oder Mindeststandards und durch die Etablierung von Monitoring-Verfahren die Funktionen der transnationalen Verbandsebene erweitert. Dies gilt mit Abstufungen mittlerweile für die Mehrzahl der Verbände. Bei einzelnen europäischen Föderationen, wie dem EMB oder der EFBH, sind darüber hinaus (zumindest was die Formalisierung und Institutionalisierung der Bearbeitung lohnpolitischer Interdependenzen anbetrifft) Entwicklungstendenzen hin zu einer genuin transnationalen Steuerung vorhanden.

Die branchenbezogene transnationale Tarifkoordination bleibt freilich mit Problemen behaftet, die diesen Ansatz ,transnationaler gewerkschaftlicher Selbsthilfe' dauerhaft labil machen: Dazu zählen zunächst strukturelle Faktoren wie die unterschiedlichen nationalen Tarifvertragsebenen und die nach Ländern enorm variierenden tariflichen Deckungsraten. Dazu zählen des Weiteren binnenorganisatorische Faktoren, die sich aus dem rein voluntaristischen Charakter der von den europäischen Gewerkschaftsverbänden verfolgten Koordinierungsansätze ergeben, da die europäische Verbandsebene nicht über die politische Autorität verfügt, die nationalen Mitgliedsorganisationen auf die Einhaltung der Koordinierungsregeln zu verpflichten.

Schließlich ist der Erfolg des transnationalen Koordinierungsansatzes in starkem Maße von machtpolitischen Faktoren abhängig, denn selbst wenn die nationalen Mitgliedsorganisationen gewillt sind, die europäischen Koordinierungsregeln zu befolgen, entscheidet letztlich ihre Stärke in den nationalen Tarifverhandlungen darüber, ob sie die transnational vereinbarten Ziele realisieren können. Angesichts eines in zahlreichen europäischen Ländern voranschreitenden Mitglieder- und damit Machtverlustes der Gewerkschaften bleibt dies eine Achillesferse jedweden transnationalen Politikansatzes.

Schließlich sind im Handlungsfeld der Konzernpolitik annähernd allen europäischen Gewerkschaftsverbänden neue Aufgaben zugewachsen. Sie erfüllen - wenngleich in unterschiedlicher zeitlicher Entwicklung und bei unterschiedlichen Intensitätsgraden - spezifische Funktionen bei der Verhandlung, Unterstützung und Praxisentwicklung von EBR. Der EBR-Prozess führte dabei einerseits zu einem veränderten und erweiterten Aufgabenspektrum der Sekretariate und ging andererseits bei einer Reihe von Verbänden mit dem Aufbau interner grenzübergreifender Arbeitsstrukturen in Form von konzernpolitischen Ausschüssen, Arbeitsgruppen oder Netzwerken einher.

Mit der Entwicklung des Handlungsfelds der EBR haben die europäischen Gewerkschaftsverbände sowohl beim Top-Management europäischer Unternehmen und bei den politischen Organen der Europäischen Union als auch bei ihren nationalen Mitgliedsgewerkschaften eine Aufwertung erfahren. Insbesondere in der Frühphase des EBR-Prozesses waren die Generalsekretäre oder Sekretariatsmitarbeiter der europäischen Gewerkschaftsverbände unmittelbar an den EBR-Verhandlungsprozessen beteiligt; zum Teil als direkte 
Verhandlungsführer, zum Teil als Koordinatoren der nationalen betrieblichen und gewerkschaftlichen Verhandlungsgruppen.

Mit dem zahlenmäßigen Wachstum der EBR und der nach der EBR-Richtlinie rechtlich vorstrukturierten Verhandlungsprozedur (der ab 1996 begonnenen sogenannten Artikelsechs-Phase) hat sich die Verhandlungsrolle und die Steuerung der Verhandlungsprozesse weitgehend auf die Ebene der nationalen Mitgliedsverbände (zurück-)verlagert. Das heißt, diese Entwicklung ist neben den Kapazitätsgrenzen der transnationalen Verbände vor allem dem besonderen Vereinbarungsmodus der Artikel-sechs-Phase geschuldet, wonach ein , besonderes Verhandlungsgremium' (nationaler) Belegschaftsvertreter beziehungsweise Gewerkschaften die EBR-Einrichtung mit dem Konzernmanagement aushandelt.

Unterschiedliche und nach Verbänden variierende Kompetenzzuschneidungen zwischen der nationalen und europäischen Gewerkschaftsebene kennzeichnen die Entwicklung des konzernpolitischen Handlungsfeldes seit Ende der 1990er Jahre. Mehrheitlich erfüllen die europäischen Gewerkschaftsverbände Orientierungsfunktionen und Unterstützungsaufgaben bei der Einrichtung und Betreuung von EBR mittels Konzerndatenbanken, der Dokumentation von Fällen guter EBR-Praxis und der Erstellung von EBR-Arbeitshilfen. Darüber hinaus sind einzelne europäische Gewerkschaftsverbände der ,Ort ${ }^{\star}$ einer durch die Verbandsexekutiven beschlossenen Steuerung der dezentralen Verhandlungs- und Betreuungsaktivitäten in Form von inhaltlichen und prozeduralen Leitlinien. Allerdings verfügen die europäischen Gewerkschaftsverbände, vergleichbar dem Handlungsfeld der tarifpolitischen Koordinierung, über keinen Durchsetzungs- und Sanktionsmechanismus im Falle der Nichteinhaltung der Leitlinien durch einzelne Mitgliedsgewerkschaften.

Die ,überschießende Praxis` einer zunehmenden Zahl von EBR (unter den gegenwärtig existierenden rund 900 EBR), die über die in der EBR-Richtlinie festgelegten Funktionen der Information und Konsultation hinausgreift und zu transnationalen Konzernvereinbarungen führt, sowie die Zunahme von Konflikten im Zuge europäischer Unternehmensumstrukturierungen haben bei einzelnen europäischen Gewerkschaftsverbänden, darunter dem , Vorreiter' EMB, inzwischen zu weitergehenden Handlungsansätzen geführt. Diese zielen zum einen auf eine noch engere europäische Koordinierung der nationalen gewerkschaftlichen Unternehmenspolitiken und zum anderen auf die Entwicklung und Durchsetzung eines transnationalen Verhandlungsverfahrens, das die verhandelnden nationalen Gewerkschaften und EBR an die europäische Verbandsebene zurückbinden soll. Ob sich dieser bislang nur in wenigen Fällen erprobte Ansatz, der den europäischen Verband zur zentralen Steuerungsebene macht, insgesamt durchsetzt und auch in den anderen europäischen Gewerkschaftsverbänden mittelfristig Nachahmung findet, bleibt abzuwarten. Denn noch ist nicht abschlieBend geklärt, welche Akteure (Gewerkschafts- oder betriebliche Arbeitnehmervertreter) auf welcher Ebene (national oder europäisch) legitimiert sind, europäische Unternehmensverhandlungen zu führen. ${ }^{20}$

\section{Zusammenfassung: verdichtete transnationale Interaktion und graduelle Profilstär- kung der europäischen Gewerkschaftsverbände}

Eine Gesamtbetrachtung der Entwicklung der zwölf europäischen Gewerkschaftsverbände ergibt ein vielschichtiges Bild. Gleichwohl lassen sich einige zentrale Entwicklungs-

20 Hans-Wolfgang Platzer/Torsten Müller/Stefan Rüb: Transnationale Vereinbarungspolitik auf Unternehmensebene. Akteurskonstellationen, Konfliktstrukturen und Entwicklungstendenzen im Verhältnis betrieblicher und überbetrieblicher Interessenvertretung in einem transnationalen Mehrebenensystem der Arbeitsbeziehungen, unveröffentlichter Projektantrag bei der Hans-Böckler-Stiftung 2008 eingereicht und seit 2009 gefördert. 
muster und -trends im Wandel der Funktionsprofile mehr oder weniger aller Verbände identifizieren und abschließend wie folgt verallgemeinern:

Im Vergleich zu den Profilen der transnationalen Verbände in den ersten Jahrzehnten nach Gründung der Integrationsgemeinschaft, die meist den Charakter von runden Tischen und Foren hatten (wobei zudem in einzelnen Branchen noch keine vom globalen Verband unabhängigen europäischen Organisationsstrukturen existierten), haben sich die Interaktionen innerhalb der transnationalen Verbände seit den 1990er Jahren sukzessive verdichtet, thematisch erweitert und politisch intensiviert. Diese schrittweise Vertiefung der transnationalen Zusammenarbeit in den oben beschriebenen Kernbereichen gewerkschaftlicher Branchenpolitik vollzog sich ungeachtet der Probleme und Herausforderungen, die mit der Erweiterung des Mitgliederkreises im Zuge der EU-Osterweiterung und der damit einhergehenden Heterogenisierung der mitgliedschaftlichen Interessen verbunden waren.

In den vergangenen zwei Dekaden haben sich neben der Fortschreibung von Verbandsroutinen im Bereich der mitgliederbezogenen Politik der europäischen Gewerkschaftsverbände (Öffentlichkeitsarbeit, Informationsdienstleistungen, Schulungsaktivitäten etc.) mit der Ausbildung neuer transnationaler Handlungsfelder und -instrumente zugleich die Funktionsprofile der Verbände verändert. Insbesondere in den Bereichen der Tarifpolitik, der Konzernpolitik und der arbeitspolitischen Selbstregulierung im Rahmen der Sektoralen Sozialdialoge nutzen die Mitgliedsverbände den transnationalen Organisationsrahmen als ,Ort ${ }^{\text {* }}$ einer zunehmend verbindlicheren und thematisch konkreteren Interessenabstimmung und Handlungskoordination, um im Mehrebenensystem der Europäischen Union und im einheitlichen Wirtschafts- und Währungsraum Interdependenzprobleme zu bearbeiten.

Eine zentrale Triebfeder der graduellen Erweiterung und Stärkung der Funktionsprofile der europäischen Gewerkschaftsverbände waren mithin die mit der Vollendung des Binnenmarktes und der Europäischen Währungsunion verbundenen Herausforderungen und Notwendigkeiten einer intensivierten europäischen Koordinierung und verbindlicheren Steuerung auf transnationaler Verbandsebene. Weitere externe Impulse, die die transnationale Zusammenarbeit der Gewerkschaften beförderten, gingen von verschiedenen seit Beginn der 1990er Jahre intensivierten Aktivitäten und politischen Weichenstellungen der EUKommission aus. Dazu zählen die 2002 vollzogene industriepolitische Neuorientierung der EU-Kommission mit einer stärkeren Betonung sektoraler Maßnahmen und der Schaffung tripartistischer Dialog- und Beratungsforen, die die direkten Beteiligungsmöglichkeiten der europäischen Gewerkschaftsverbände an der Formulierung und Umsetzung industriepolitischer Maßnahmen verbesserte. Dazu zählt ferner die 1998 durchgeführte Reform des Sektoralen Sozialdialogs, die durch die institutionelle Förderung der Einrichtung von ASSD den Auf- und Ausbau von Dialog- und Arbeitsbeziehungen der europäischen Gewerkschaftsverbände mit europäischen Branchenverbänden der Arbeitgeberseite erleichterte. Dazu zählt schließlich im Handlungsfeld ,Unternehmenspolitik' die Verabschiedung der EBR-Richtlinie im September 1994, die den Aufbau von Dialog- und Verhandlungsbeziehungen zur Arbeitgeberseite auf Unternehmensebene erleichterte und darüber hinaus gewerkschaftsintern eine wichtige institutionelle Voraussetzung zur Entwicklung einer europäisch koordinierten Unternehmenspolitik schuf.

Auch wenn somit die Gesamtentwicklung während der vergangenen zwei Integrationsdekaden in Richtung einer sukzessiven Erweiterung und graduellen Stärkung der Funktionsprofile europäischer Gewerkschaftsverbände verweist, bleiben - gemessen an den Herausforderungen eines transnationalen EU-Wirtschafts- und Währungsraumes - bei allen Verbänden neben der Ressourcenschwäche auch zahlreiche politische Steuerungsprobleme virulent. Dazu zählen nicht zuletzt die Widersprüche zwischen ,Deklaration“ und ,Praxis‘. 
Dies bedeutet, dass die Verständigungsprozesse, etwa im Bereich der Tarif- und Konzernpolitik, zwar zur Festlegung, verbindlicher' Ziele und Verfahrensregeln auf europäischer Ebene führen, die Einhaltung der europäisch vereinbarten Politiken aber letztlich von der Selbstbindung und Selbstverpflichtung der Mitgliedsverbände und deren dezentraler Durchsetzungsmacht in den jeweils nationalen Kontexten abhängig bleibt.

\section{Schriftenreihe des AEI e.V.}

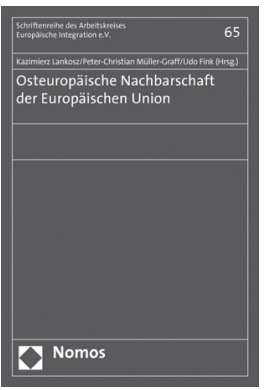

\section{Osteuropäische Nachbarschaft der Europäischen Union}

Herausgegeben von Prof. Dr. Dr. h.c. Kazimierz Lankosz, Prof. Dr. Dres. h.c. Peter-Christian Müller-Graff und Prof. Dr. Udo Fink

2009, Band 65, 129 S., brosch., 24,- $€$, ISBN 978-3-8329-4791-O

Die Nachbarschaft der Europäischen Union erstreckt sich im Osten von der Barents-See bis zum Schwarzen Meer und umfasst dabei gänzlich unterschiedlich strukturierte Staaten. Der Band enthält Beiträge zu Grundfragen, Einzelfragen und konzeptionellen Perspektivfragen bezüglich des nachbarschaftlichen Verhältnisses der EU zu diesen Staaten, insbesondere zur Ukraine sowie zu Moldawien und Georgien.

Bitte bestellen Sie im Buchhandel oder versandkostenfrei unter $\downarrow$ www.nomos-shop.de

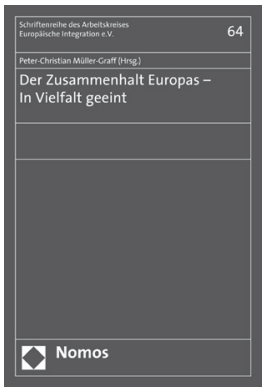

\section{Der Zusammenhalt Europas - In Vielfalt geeint}

Herausgegeben von Prof. Dr. Dres. h.c. Peter-Christian Müller-Graff

2009, Band 64, 137 S., brosch., 29,- $€$, ISBN 978-3-8329-4839-9

Der Zusammenhalt der aus 27 Mitgliedstaaten bestehenden Europäischen Union kann nicht allein durch das europäische Primärrecht als konsentiertes normatives Gehäuse gewährleistet werden. Darüber hinaus sind viele zusätzliche Realelemente eines förderlichen Kontexts erforderlich. Wesentliche Kernelemente aus deren Kreis sind Gegenstand der Beiträge dieses Werkes. 